\title{
A Review on Tip Clearance Flow and Secondary Flow Losses in Linear Turbine Cascade
}

\author{
U.J.Patdiwala ${ }^{1}$, H.C.Patel ${ }^{2}$, Paresh K. Parmar $^{3}$ \\ ${ }^{I}$ (Assistant Professor, Mechanical Engg. Dept., Gandhinagar Institute of Technology, India) \\ ${ }^{2}$ (Assistant Professor, Mechanical Engineering Dept., S.R.Patel Engg. College ,Unja, India) \\ ${ }^{3}$ (M.E. Research Scholar, Mechanical Engg. Dept., Gandhinagar Institute of Technology, India)
}

\begin{abstract}
The aerodynamics of the flow in a turbine stage (stator/rotor) is rather complex and still subject of many ongoing research activities in the gas turbine community. Gas turbine have air is the most important fuel which is produce power. It is easily and freely available in nature. But in the gas turbine have many losses occurs in running condition of it. The cascade arrangement has different-different type aerodynamic losses occurring in turbine blade. In the turbine blade have occurred profile loss, annulus loss, secondary loss and tip clearance flow loss. In this all losses the tip clearance flow and secondary flow losses have major effect in the turbine efficiency. So the turbine efficiency improvement kindly depends on these losses. The knowledge of these losses provides better idea to reduce these losses. This article provides review on different studies on secondary flow and tip clearance flow losses in linear turbine cascade.
\end{abstract}

Keywords: Tip clearance flow, secondary flow, and linear turbine cascade.

\section{Introduction}

The efficiency of gas-turbine engines is an ongoing concern for both the designers and operators of these devices. High fuel costs and the broad awareness of finite oil resources have prompted a greater interest towards reducing the fuel usage, and thus, the aerodynamic inefficiencies that are present within turbo machines. Inefficiency within a turbine stage accumulates due to aerodynamic losses from a variety of sources. Overall, the largest single source of loss is caused by the tip leakage flow. In both the compressor and turbine stages of unshrouded gas turbine engines, the rotation of the blade rows with respect to the stationary casing, or end wall, necessitates having a small tip clearance from the casing. The leakage of flow through this tip clearance causes a number of adverse effects that result in a reduction in the overall efficiency of a turbine stage. It has been stated that the loss in turbine stage efficiency due solely to the tip clearance flow varies between $2 \%$ to $6 \%$ for a realistic clearance of $1 \%$ of blade span, and that this loss accounts for $20 \%$ to $40 \%$ of the overall loss within the turbine [1].The potential savings of reducing this unwanted loss is great. Lattime and Steinetz [2] reported that for only a $1 \%$ reduction in specific fuel consumption, the potential fuel savings for commercial U.S. airline carriers alone would be approximately 53 billion liters (14 billion gallons) annually. From the results of Lattime and Steinetz, as well as this qualitative analysis, even a small reduction in the flow penalty would yield a large improvement in emissions and cost savings. Turbo machines have a small clearance, referred to as the tip clearance or tip gap, between outer radius of the rotating blades and stationary casing. Aerodynamic losses are generated from a number of sources. Denton states that losses are typically broken down into profile loss, end wall loss, and leakage loss [3]. Profile loss is loss generated in the blade boundary layers and blade wakes. End wall loss refers to loss that is a result of the end wall boundary layer passing through the tip clearance. Leakage loss pertains to losses incurred by the flow that passes through the blade tip clearance. The leakage flow through the clearance causes both end wall loss and leakage loss. It is the loss of flow through the tip clearance from the main passage and the subsequent irreversible mixing and diffusion that are the sources of inefficiency. The presence of the blade tip clearance will cause less-than-ideal conversion of the available total enthalpy into rotational motion. The turbine tip clearance allows air to leak over the blade tip and no work can be extracted from this fluid. Because of the large extent to which the different loss mechanisms interact, they are not easily separable from each other. There are generally three types of vortex occurred in the turbine: the tip leakage vortex, the horseshoe vortex, and the passage (or cross flow) vortex. It is known that the leakage vortex is in close proximity to the passage and horseshoe vortices, allowing for interaction and mixing between all three structures. Therefore the leakage flow and tip leakage vortex cannot be studied in isolation. Other nearby vortical flows, in addition to the leakage vortex, must be examined, understood, and included in the analysis, as the leakage flow interacts highly with them.

\section{1 losses in turbine}

Principle aerodynamics losses occurring in most of the turbo machines arise due to the growth of the boundary layer and its separation on the blade and passage surfaces. Other occurs due to wasteful circulatory 
flows and the formation of shock waves. Non-uniform velocity profiles at the exit of the cascade lead to another type of loss referred to as the mixing or equalization loss. Aerodynamic losses occurring in a turbine blade cascade can be following types:

\subsubsection{Profile Loss}

This loss is associated with the growth of the boundary layer which is directly related to blade profile. When the adverse pressure gradient on the surfaces so this increase profile losses by the separation of boundary layer. The pattern of the boundary layer growth and its separation depend on the geometries of the blade and flow. The suction side of a blade is more prone to boundary layer separation than pressure side of blade. So the suction side aerodynamic losses higher than pressure side of blade surfaces.

\subsubsection{Annulus Loss}

These losses generally occur in the rotating row of blades. The majority of blade rows in turbo machines are housed in casings. The axial turbine stage has a pair of fixed and moving blade rows. This pair of blade at end wall becomes occur this losses.

\subsubsection{Secondary Flow Loss}

This loss occurs in the regions of flow near the end walls owing to the presence of unwanted circulatory or cross flows. The flow near the end walls give rise to circulatory flows which are mixes with main flow through the blade passage. As a result of this, secondary vortices in the stream wise direction are generated in the blade passages. These vortices try to transport low energy fluid from the pressure side to suction side of the blade passage, thus increasing the possibility of separation of the boundary layer on the suction side. It is observing that the secondary flow can also affect the profile and annulus losses.
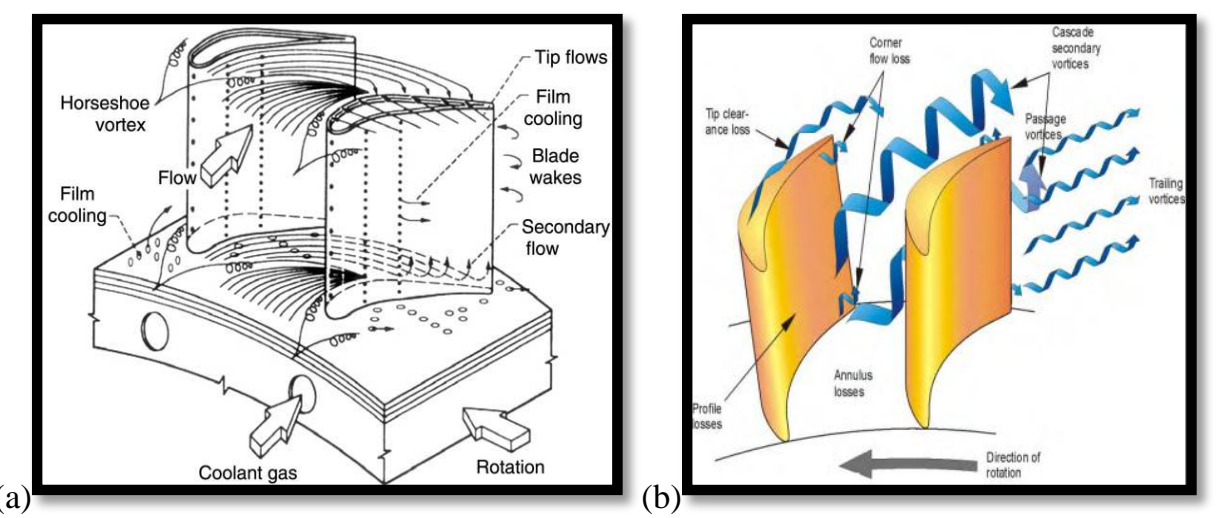

Fig. 1. Illustration of turbine losses (a) secondary flow loss and (b) Tip clearance flow loss.

\subsubsection{Tip Clearance Flow Loss}

These losses arise due to the clearance between a moving blade and the casing. On account of the static pressure difference, the flow leaks from the pressure side towards to suction side. The tip clearance and secondary flows are closely related to each other and it is often convenient to estimate them together.

\section{Tip clearance flow}

\section{Literature Review}

Venkateswara Babu, N Sitaram, M Govardhan [4], studied experimental investigation of tip clearance effects on flow field in an annular turbine cascade. The experiment take on inlet, in the blade passage, and exit of the cascade using five hole probe and seven hole probe. The author found that secondary and tip clearance losses steeply increase lower side of the blade trailing edge indicating additional losses due to vortex and clearance flow mixing with the main flow. And all this occurred finally losses in linear turbine cascade so the efficiency lowers down. Topology and vortex structures of turbine cascade with different tip clearance studied by Han Warrjin, Yang Qinghai, Huang Hongyan, and Jia Lin [5]. They found that the different-different clearances the blade to eliminate the upper passage vortex, waken the blade tip separation and thus reduces the relative leakage and energy loss. Topology and vortex structures of the cascade wall surface flow changes than vortex intensity, scale and its partnering effect, increases so loss of energy in flow. Unsteady loss in a high pressure turbine stage studied by S.J.Payne, R.W. Moss, and N.W.Harvey [6]. They measure four major loss mechanisms: the tip leakage vortex, the upper passage vortex, the wake and the lower passage vortex. They 
finally found that the tip leakage vortex have major entropy generation than other losses. Heat transfer of a gas turbine blade have effect of rim location, rim height and tip clearance on tip etc. studied by Jae Su Kwak, Jaeyong Ahn, Je-chin Han[7]. By using a rimmed tip blade, the heat transfer coefficients on the blade tip and shroud were reduced significantly. They observed that the rim height increased the heat transfer coefficient on the tip decreased. On the pressure side, the effects of the rim height, rim location, and tip gap clearance on the heat transfer coefficient were not significant.

Tip clearance flow in turbine cascades studied by Li Wei Qiao and Weiyang [8]. They studied that the measure of total pressure loss coefficient are made at cascade outlets b using five hole probe different exit mach number and different tip clearance height. The author studied at exit mach numbers of $0.10,0.14$, and 0.19 than different tip clearance heights of $1 \%, 1.5 \%, 2 \%, 2.5 \%$, and $3 \%$ of the blade height. They found that increased tip clearance heights in the tip regions of two adjacent blades are blame for the larger clearance loss of the centre blade. Effects on turbine tip clearance on gas turbine performance studied by Cleverson Bringhenti and Joao Roberto Barbosa [9]. They observed that the tip clearance have very well affect on its performance and occurs losses. They also found that the turbine tip clearance effect also seen by the compressor characteristics and the engine performance. And this information used during early design phase to take into account of turbine tip clearance losses. Tip leakage flows in axial turbine have active control on it simulated by Li Wei, Qiao Weiyang, Xu Kaifu and Luo Hualing [10]. They use for numerical simulation have CFD code integrated with dense-correction-based 3D Reynolds-averaged Navier-stoke equations with the well proven Reynolds stress model used. They conclude that blockage on the tip clearance flow thus decreasing the tip leakage loss. The loss region tip clearance and tight tip clearance increase the efficiency $0.35 \%$ and $0.30 \%$ respectively. So generally clearance has effective to its efficiency. Tip injection on tip clearance flow in an axial turbine cascade has both the numerical and experimental studied by Maosheng Niu a, Shusheng Zang [11]. They studied that tip injection can weaken tip clearance flow, reducing the tip clearance mass flow and its associated losses. They found that injection chord wise location plays an important role in the redistribution of secondary flow within the cascade passage. Gas turbine characteristics have varying tip clearance and axial gap investigated by Rajesh Yadav, Vishal Gulati and Puneet Katyal [12]. They conclude that axial gap of $3.5 \mathrm{~mm}$ and 5\% tip clearance is the optimum set value for maximum performance.

\section{Secondary flow}

Kristina S. Herman son and Karen A. Thole [13] studied the effect of Mach number on secondary flow characteristics. They use the low speed wind tunnel for experimental procedure and also compare secondary flow at low and high Mach number conditions. They found that downstream of the trailing edge the relative magnitude to stream wise and secondary velocities reduces the flow deviations for the high Mach number condition. Total pressure loss at suction side higher than pressure side. The secondary flow vortex structure has effect of wake distribution in a turbine blade cascade studied by Christopher G. Murawski and Kambiz Vafai [14]. They use three different Reynolds number like 25000, 50000, and 85000 and velocity of wake generator was varied from $1 \mathrm{~m} / \mathrm{s}$ to $4.8 \mathrm{~m} / \mathrm{s}$. They found that the secondary flows create the second greatest profile loss in the high pressure turbine stage and low pressure turbines contain blades with larger aspect ratio, so low pressure turbine stage secondary flows have a lesser affect on total profile loss. All the path of turbine blades wake passing frequency to axial chord flow frequency greater than 1, therefore the secondary flow not induces between wake distributions.CFD analysis of secondary flows and pressure losses in a NASA transonic turbine cascade studied by Valery Goriatchev, Nikolay Ivanov, Evgueni Smirked, Vladimir Ris[15]. They use software like FLUENT 5.5, SINF. And also use an advanced post-processing tool for flow visualization. They found that the streamline patterns presented help one to get a more insight into the feature of secondary flows developing in turbine blade cascades with large turning angle.

CFD analysis of 3D flow structure and end wall heat transfer in a transonic turbine blade cascade studied by Alexander M. Levchey and Evgueni M. Smirnov [16] and also studied by effects of grid refinement. They use the MSST model for CFD analysis. This is the best model for CFD analysis. With CFD codes of second-order accuracy, one should use grids comprised of about or more than 2 million cells (for each full blade passage) to get a definite conclusion on preference of one or another turbulence model for prediction of secondary as well as tip clearance flow phenomenon under consideration. Computational study of Mach number effects on secondary flows in a linear turbine cascade studied by M.Mahendran and N.Sitaram [17]. They found that the Mach number increases at axially than total pressure losses decreases at desire level and when velocity increases the total pressure losses decreases. This study enhances our knowledge of secondary flows for the future turbine design. Stream wise vortex have used to control secondary flow in cascade studied by Qi Lei, Zou Zhengping, Wang Peng, Cao tens[18]. They found that stream wise vortex can effectively reduce the stagnation pressure loss and flow deviation. The vortex generator provides stream wise vortex in turbine. Both the experimental and computational results in this configuration show a decrease in stagnation pressure loss at the cascade exit by $5.5 \%$ and $7.5 \%$. Radial pressure gradient have effect on the secondary flow generated in an 
annular turbine cascade studied by Hesham M. El-batsh [19]. They studied that the secondary losses becomes higher at hub side and lower at shroud side of cascade arrangement. So finally they found that the secondary flow loss reduction have hub side create passage vortex and try to reduce this secondary flow losses.

The geometrical designed four turbine blade cascade behind secondary flow losses studied by Ali S. Abas and A.I.Noor Y.Abas[20]. They use mathematical techniques like Rapid Axial Turbine Design (RATD). In this study they use rectangular wind tunnel and four turbine blade cascade arrangement fix in it. They found that the maximum static loss regions becomes all downstream axial distance of passage vortex, trailing edge vortices etc. And this is maximum static loss in turbine cascade exit side. Turbine blade tip shape effect on total pressure loss and secondary flow of a linear turbine cascade studied by Young Cheol Nho, Jung Ship Park, Young Jin Lee, and Jae Su Kwak [21]. They studied at different-different tips are: plane, single or double squealer, dimpled, chamfered, and grooved tips at different tip clearance of $1.5 \%$ and $2.3 \%$ of the blade span were tested. The entire tested tip shapes the double squealer tip and the grooved along pressure side tip performed best and both tip showed smaller total pressure loss coefficient than that of the pane tip. Secondary flows have effect on heat transfer of a gas turbine blade studied by Hesham M. El-Batish, Sawieh A. Nada, Samia Nasreldin Abdo, and Abdelgolil A. El-Tayesh [22]. The two dimensional research have done but three dimensional heat transfer measurement not taken well. So heat transfer measurement and numerical calculations were performed in this study. They found that the secondary flow region higher on suction side of blade so the heat transfer higher at this side.

\section{Conclusion}

Based on the review the following conclusions have been drawn.

- The leakage vortex introduces a characteristic overturning and underturning of the near end wall flow with respect to the main passage flow.

- The leakage flow is a function of the mass flow through the clearance, which is in turn dependent on the tip clearance gap height.

- The fluid near the blade tip is strongly accelerated around the pressure side corner into the clearance.

- Most of the clearance mass flow occurs in the half of the clearance height to the end wall, while most of the tip clearance total pressure loss takes place near the tip surface.

- Secondary flow is highly three-dimensional and undesirable flow occurs due to strong interaction of boundary layers ahead of the turbine blade leading edge.

- Tip clearance flow occurs due to tip gap between turbine blade and end-wall of the cascade, which results into highly deflection/turning of the flow beneath the turbine blade and it leaks through pressure side to suction side.

- Both this phenomena of secondary flow and tip clearance flow are responsible for generating low pressure region towards the blade trailing edge and also results into reduction of efficiency of the turbine.

- As the blade loading increases the turbine aerodynamic performance decreases and it's mainly due to these two losses, which take place in turbine cascade.

- Certain methods can be adopted in order to reduce these secondary and tip clearance losses by analysis flow structure around the blade leading edge, trailing edge and in the passage.

\section{References}

[1] B. Lakshminarayana. "Fluid Dynamics and Heat Transfer of Turbo Machinery". John Wiley \& Sons, Inc., 1996. New York.

[2] S. B. Lattime and B. M. Steinetz. "High-Pressure-Turbine Clearance Control Systems: Current Practices and Future Directions", 2004, Aiaa J. Of Propulsion and Power, 20(2):302-311.

[3] J. D. Denton. "Loss Mechanisms in Turbo Machines". Asme J. Of Turbo Machinery, 115:621-656, October 1993. 93-Gt-435

[4] Venkateswara Babu, N Sitaram , M Govardhan, 2002, "Experimental investigation of tip clearance effects on flow field in an annular turbine rotor cascade", pp, 424-431

[5] Han Warrjin, Yang Qinghai, Huang Hongyan, Jia Lin, 2002, "Topology and vortex structures of turbine cascade with different tip clearance".

[6] S.J.Payne, R.W. Moss, and N.W.Harvey, 2003, "unsteady loss in a high pressure turbine stage", 698-708.

[7] Jae Su Kwak, Jaeyong Ahn, Je-chin Han, 2004, "Heat transfer of a gas turbine blade have effect of rim location, rim height and tip clearance on tip", 5651-5663.

[8] Li Wei Qiao and Weiyand, 2008, “Tip clearance flow in turbine cascades”, Chinese Journal of Aeronautics 21, 193-199

[9] Cleverson Bringhenti and Joao Roberto Barbosa,2008, "Effects on turbine tip clearance on gas turbine performance"

[10] Li Wei, Qiao Weiyang, Xu Kaifu and Luo Hualing,2009, "Tip leakage flow in axial turbine have active control on it simulated", Chinese Journal of Aeronautics 22, 129-137

[11] Maosheng Niu a, Shusheng Zang, 2011, “. Tip injection on tip clearance flow in an axial turbine cascade has both the numerical and experimental investigation" Experimental Thermal and Fluid Science 35,1214-1222

[12] Rajesh Yadav, Vishal Gulati and Puneet Katyal, 2011, "Investigation of gas turbine characteristics by varying tip clearance and axial gap", pp, 1058-1064

[13] Kristina S. Herman son and Karen A Thole, 2000, “the effect of Mach number on secondary flow characteristics”, pp, 179-196 
[14] Christopher G. Murawski and Kambiz Vafai , 2000, "The secondary flow vortex structure has effect of wake distribution in a turbine blade cascade"

[15] Valery Goriatchev, Nikolay Ivanov, Evgueni Smirked, Vladimir Ris, 2003, "CFD analysis of secondary flows and pressure losses in a NASA transonic turbine cascade"

[16] Alexander M. Levchey and Evgueni M. Smirnov, 2007, "CFD analysis of 3D flow structure and end wall heat transfer in a transonic turbine blade cascade", 19-22.

[17] M.Mahendran and N.Sitaram, 2007, "Computational study of mach number effects on secondary flows in a linear turbine cascade"

[18] Qi Lei, Zou Zhengping, Wang Peng, Cao tens, 2012, "Stream wise vortex have used to control secondary flow in cascade", Computers \& Fluids 54, 45-55

[19] Hesham M. El-batsh, 2012, "Radial pressure gradient effect on the secondary flow generated in an annular turbine cascade".

[20] Ali S. Abas and A.I.Noor Y.Abas, 2012, "A geometrical designed four turbine blade cascade behind secondary flow losses".

[21] Young Cheol Nho, Jung Ship Park, Young Jin Lee, Jae Su Kwak, 2012, "Turbine blade tip shape effect on total pressure loss and secondary flow of a linear turbine cascade", International Journal of Heat and Fluid Flow 33, 92-100.

[22] Hesham M. El-Batish, Sawieh A. Nada, Samia Nasreldin Abdo, and Abdelgolil A. El-Tayesh, 2013, "Secondary flows have effect on heat transfer of a gas turbine blade 